\title{
Impact of exercise on articular cartilage in people at risk of, or with established, knee osteoarthritis: a systematic review of randomised controlled trials
}

\author{
Alessio Bricca, ${ }_{1}^{1}$ Carsten B Juhl, ${ }_{1}^{1,2}$ Martijn Steultjens, ${ }^{3}$ Wolfgang Wirth, ${ }^{4,5}$ \\ Ewa M Roos ${ }^{\oplus 1}$
}

- Additional material is published online only. To view please visit the journal online (http://dx.doi.org/10.1136/ bjsports-2017-098661).

${ }^{1}$ University of Southern Denmark, Odense, Denmark ${ }^{2}$ Herlev and Gentofte Hospital, University of Copenhagen

Copenhagen, Denmark ${ }^{3}$ Glasgow Caledonian University, Glasgow, UK

${ }^{4}$ Paracelsus Medical University (PMU) Salzburg and Nuremberg, Salzburg, Austria

${ }^{5}$ Medical Data Processing,

Chondrometrics GmbH, Ainring, Germany

\section{Correspondence to}

Dr Alessio Bricca, University

of Southern Denmark, Odense

5230, Denmark;

abricca@health.sdu.dk

Received 10 October 2017

Revised 17 May 2018

Accepted 4 June 2018

Published Online First

22 June 2018
Check for updates

To cite: Bricca $A$, Juhl CB, Steultjens $M$, et al. Br J Sports Med 2019:53:940-947.

\section{ABSTRACT}

Objective To investigate the impact of knee joint loading exercise on articular cartilage in people at risk of, or with established, knee osteoarthritis (OA) by conducting a systematic review of randomised controlled trials (RCTs).

Design We followed the Preferred Reporting Items for Systematic Reviews and Meta-analyses guidelines.

Data sources We performed a literature search with no restriction on publication year or language in MEDLINE, EMBASE, CINAHL, the Cochrane Central Register of Controlled Trials and Web of Science up to September 2017.

Eligibility criteria RCTs investigating the impact of exercise on MRI-assessed articular cartilage in people over 18 years of age.

Results We included nine trials, including a total of 14 comparisons of cartilage morphometry, morphology and composition outcomes, of which two included participants at increased risk of knee OA and 12 included participants with knee OA. In participants at increased risk, one study comparison reported no effect on cartilage defects and one had positive effects on glycosaminoglycans (GAG). In participants with OA, six study comparisons reported no effect on cartilage thickness, volume or defects; one reported a negative effect and one no effect on GAG; two reported a positive effect and two no effect on collagen.

Conclusions Knee joint loading exercise seems to not be harmful for articular cartilage in people at increased risk of, or with, knee OA. However, the quality of evidence was low, including some interventions studying activities considered outside the therapeutic loading spectrum to promote cartilage health.

\section{INTRODUCTION}

Knee osteoarthritis (OA) is the most common joint disease and a major cause of disability and pain. ${ }^{1}$ The OA prevalence has doubled since the mid-20th century $^{2}$ with an expected higher incidence in the future. ${ }^{3}$ The annual total medical cost per person suffering from OA is on average $€ 11100$. ${ }^{4}$

Articular cartilage breakdown is the hallmark of OA, with aggrecan loss being an early sign of tissue degeneration. Many factors such as age, body mass index (BMI), knee injury, inflammation, sex and family history independently, and as a result of their interaction, contribute to its development and progression. ${ }^{5}$ For example, approximately every second major knee injury from sports results in OA $10-15$ years later, ${ }^{7-9}$ and it has been estimated that at least $12 \%$ of the total burden of knee OA originates from knee injury. ${ }^{10}$ Hypothetically, interventions targeting younger patients at increased risk of OA (eg, following sports injury), or in the early stages of the disease, increase the chances of slowing down articular cartilage breakdown since the integrity of the cartilage may still be intact with little or no aggrecan loss.

Therapeutic exercise is a first-line treatment in $\mathrm{OA}$ : it is safe $\mathrm{f}^{11}$ and effectively reduces pain and improves function. ${ }^{12-14}$ Less is known about the effects from therapeutic exercise on knee joint articular cartilage. However, exercise at higher doses, such as playing sports at elite level, is associated with development of OA, suggesting injury and also load in itself as being a contributing factor. ${ }^{15} 16$ The mechanical loading generated from exercise, in combination with cell biology, and in some cases inflammatory factors, may alter the function of articular cartilage. ${ }^{17}$ While there are no conclusive studies, it has been suggested that exercise may prevent or delay OA onset. ${ }^{18}$ In support of this, two cohort studies found that a moderate dose of physical activity could slow down cartilage degeneration in middle-aged individuals at early OA stages. ${ }^{19} 20$ Furthermore, initiating an accelerated and progressive weight-bearing intervention a few hours after cartilage surgery was shown to be safe for the cartilage and resulted in more favourable clinical outcomes compared with a delayed knee joint loading exercise intervention. ${ }^{21}$ Also, in patients having had meniscectomy, therapeutic exercise increased cartilage glycosaminoglycan (GAG) content. ${ }^{22}$ However, patients at risk of, or with, knee OA still often believe that exercise may wear down their knee joints, creating a barrier to exercise. $^{23}$

Systematic reviews of randomised controlled trials (RCTs) provide the highest quality of evidence for assessing effectiveness and harms of treatments. Current knowledge in this area of interest has not been summarised systematically. Therefore, we aimed to review the existing evidence regarding the impact of knee joint loading exercise on articular cartilage.

\section{METHODS}

Terminology

As defined by the authors of the original papers, participants at risk of knee OA are those with risk factors (eg, knee injury treated with or without surgery, or BMI $\left.\left(\mathrm{kg} / \mathrm{m}^{2}\right) \geq 25\right)$ associated with the 
development or progression of the disease, while participants with OA are those with a clinical diagnosis of OA (ie, according to the American College of Rheumatology criteria) with or without radiographic signs of knee OA (Kellgren-Lawrence grade $>1$ ), in the tibiofemoral and/or patellofemoral compartments of one or both knees. ${ }^{24}$

Articular cartilage outcomes assessed by MRI were classified into morphometry (ie, thickness and volume), morphology (ie, defects) or composition (ie, GAG assessed by delayed gadolinium-enhanced MRI of cartilage (dGEMRIC) and collagen assessed with T2-mapping in seven comparisons).

The term 'knee joint loading exercise' refers to the stimuli applied to the knee joint from 'exercise' or 'exercise therapy'. The term 'exercise' refers to 'physical activities, which are usually done on a regular basis with the intention of improving or maintaining physical fitness or health' and 'physical activity' refers to 'any bodily movement produced by skeletal muscles that requires energy expenditure'. The term 'exercise therapy' refers to 'a regimen or plan of physical activities designed and prescribed for specific therapeutic goals with the purpose to restore normal musculoskeletal function or to reduce pain caused by diseases or injuries'. 25

\section{Protocol}

This systematic review is reported according to the Preferred Reporting Items for Systematic Reviews and Meta-Analyses guidelines (online supplementary appendix A). Study selection, eligibility criteria, data extraction and statistical analysis were performed according to the Cochrane Collaboration guidelines ${ }^{23}$ and published in a protocol in the PROSPERO database (CRD42016039536).

\section{Eligibility criteria}

We included RCTs investigating the impact of knee joint loading exercise on articular cartilage in people over 18 years of age. Studies were excluded when no full text was available and when treatment arms involved interventions other than knee joint loading exercise that might have impacted on the articular cartilage.

\section{Literature search}

A systematic literature search was performed with no restriction on publication year or language in MEDLINE via PubMed, EMBASE via Ovid, CINAHL (including preCINAHL) via EBSCO, the Cochrane Central Register of Controlled Trials (CENTRAL) and Web of Science (WoS) up to May 2016. The search was repeated for the period from May 2016 to September 2017 in these databases to identify additional studies published before manuscript submission.

\section{Search methods and study selection}

The search was first performed in MEDLINE (online supplementary appendix B) and then customised for EMBASE, CENTRAL, WoS and CINAHL. All terms were searched, if possible, both as keywords $(\mathrm{MeSH})$ and as text words in titles and abstracts (TIAB). In MEDLINE and EMBASE, animal studies were identified and removed before screening all the studies, using a validated animal filter. $^{26}{ }^{27}$ Initially, two reviewers (AB and $\mathrm{CBJ}$ ) independently screened titles and abstracts, and all studies deemed eligible by at least one of the reviewers were checked independently in full text by the same two reviewers. In addition, reference lists from retrieved publications and systematic reviews published after January 2010 were screened. Disagreements between the two reviewers in inclusion were discussed until consensus was reached.

\section{Data collection}

A customised data extraction form was developed for each of the articular cartilage outcome categories: morphometry (ie, thickness and volume), morphology (ie, defects) or composition (ie, GAG and collagen). These outcomes were estimated from the combination of different cartilage compartments (ie, medial and lateral) when data were available. Otherwise, values from the medial and lateral values of the tibia, femur and the patella were used. Data were extracted by the first and second authors ( $\mathrm{AB}$ and $\mathrm{CBJ}$ ) from tables and graphs of published manuscripts. The following information was mandatory: authors of the study, year of publication, design of the trial, intervention characteristics, location of the trial (in the case of multicentre studies, primary investigator affiliation was applied), number of participants allocated (to the exercise and control groups, respectively), the participants' average age, average body mass index $\left(\mathrm{BMI}, \mathrm{kg} / \mathrm{m}^{2}\right)$, the duration of the study (presented in weeks) and the MRI characteristics. When several intervention groups were included in a study, the between-group difference was reported for each possible comparison. For example, when a study had two intervention groups (A and $B$ ) and one control group (C), we compared $A$ versus $C$ and $B$ versus $C$, and reported the results as two separate study comparisons. This procedure is in accordance with the Cochrane handbook. ${ }^{23}$

\section{Narrative synthesis of results}

\section{Between-group difference}

We assessed the effect of knee joint loading exercise as positive $($ ' + ') or negative ('-’) when a statistically significant $(\mathrm{p}<0.05)$ improvement or decline in the outcome of interest was reported for the overall cartilage or at least one of the cartilage compartments assessed in the intervention group compared with the control group. If none of the compartments showed an increase or a decrease in the outcome of interest, we reported this finding as no effect ('=').

Increased T2 values have been associated with deteriorated collagen orientation and increased hydration, ${ }^{28}{ }^{29}$ which is considered to have a negative impact on the cartilage. Therefore, we reported increased T2 values as negative ('-') and decreased T2 values as positive ('+') for the cartilage. A decrease in cartilage thickness/volume was interpreted as negative for the cartilage. Accordingly, an increase in cartilage thickness/volume was interpreted as potentially beneficial. However, the proof of a positive effect on cartilage volume/thickness would need additional information since increased cartilage volume/thickness may also be related to the growth of the subchondral bone for example.

\section{Within-group difference}

Additionally, we investigated within-group differences assessing the effect of knee joint loading exercise as positive ('+') or negative ('-') when an improvement or a decline in the outcome of interest was reported between preintervention and postintervention, and as no effect ('=') if none of the compartments showed an increase or a decrease in the outcome of interest.

\section{Overall quality of evidence}

Risk of bias

Study quality was assessed by rating the risk of selection bias, performance bias, detection bias, attrition bias, reporting bias 
and other sources of bias. Two reviewers (AB and $\mathrm{CBJ}$ ) independently assessed whether each of the following domains was adequate (eg, low, unclear or high risk of bias): 'sequence generation', 'allocation concealment', 'blinding', 'incomplete outcome data addressed', 'selective outcome reporting' or 'other bias' (eg, funding). ${ }^{23}$ Disagreements in initial ratings of methodological quality assessment were discussed between the two reviewers until consensus was reached.

Knee joint loading exercise quality assessment

Based on a combination of theoretical and clinical considerations, two of the authors (CBJ and EMR) independently assessed the anticipated impact of the knee joint loading interventions on cartilage (low, moderate or high) and if the dose was considered adequate to presume positive cartilage modifications were possible. High-impact activities (eg, jumping) ${ }^{30}$ and participation in sports ${ }^{15}$ is associated with cartilage deformation and increased risk of radiographic OA. Similarly, lack of knee joint loading in the form of knee immobilisation ${ }^{31}$ or sedentary behaviour ${ }^{19}{ }^{20}$ is associated with detrimental cartilage changes. Therefore, interventions including activities being considered outside the therapeutic loading spectrum were assessed as inadequate to promote cartilage health. Accordingly, the anticipated impact was considered to be too high in interventions focusing on jumping and too low in aquatic exercise.
GRADE assessment

The overall quality of evidence for the estimates was evaluated using the GRADE (Grading of Recommendations Assessment, Development and Evaluation) approach. The GRADE is a systematic approach to rate the quality of evidence across studies for specific outcomes. It is based on five domains that involve the methodological flaws of the studies (ie, risk of bias), the heterogeneity of results across studies (ie, inconsistency), the generalisability of the findings to the target population (ie, indirectness), the precision of the estimates and the risk of publication bias.

\section{RESULTS}

\section{Study selection and characteristics}

The literature search identified a total of 2868 unique publications, of which 21 individual RCTs were identified as potentially eligible. Ultimately, we included nine papers, involving 14 study comparisons (figure 1). MRI-assessed cartilage morphometry was investigated in four, ${ }^{32} 33$ cartilage morphology in three ${ }^{34-36}$ and cartilage composition in seven comparisons. ${ }^{22}$ 37-39 One study was reported in two different papers. ${ }^{3740}$ Multanen et al $l^{37}$ reported findings in the tibiofemoral compartment and Koli et $a l^{40}$ in the patellofemoral compartment of the same participants following the same exercise intervention. We included both papers and counted them as one study with two study comparisons, as suggested in the Cochrane guidelines. ${ }^{23}$
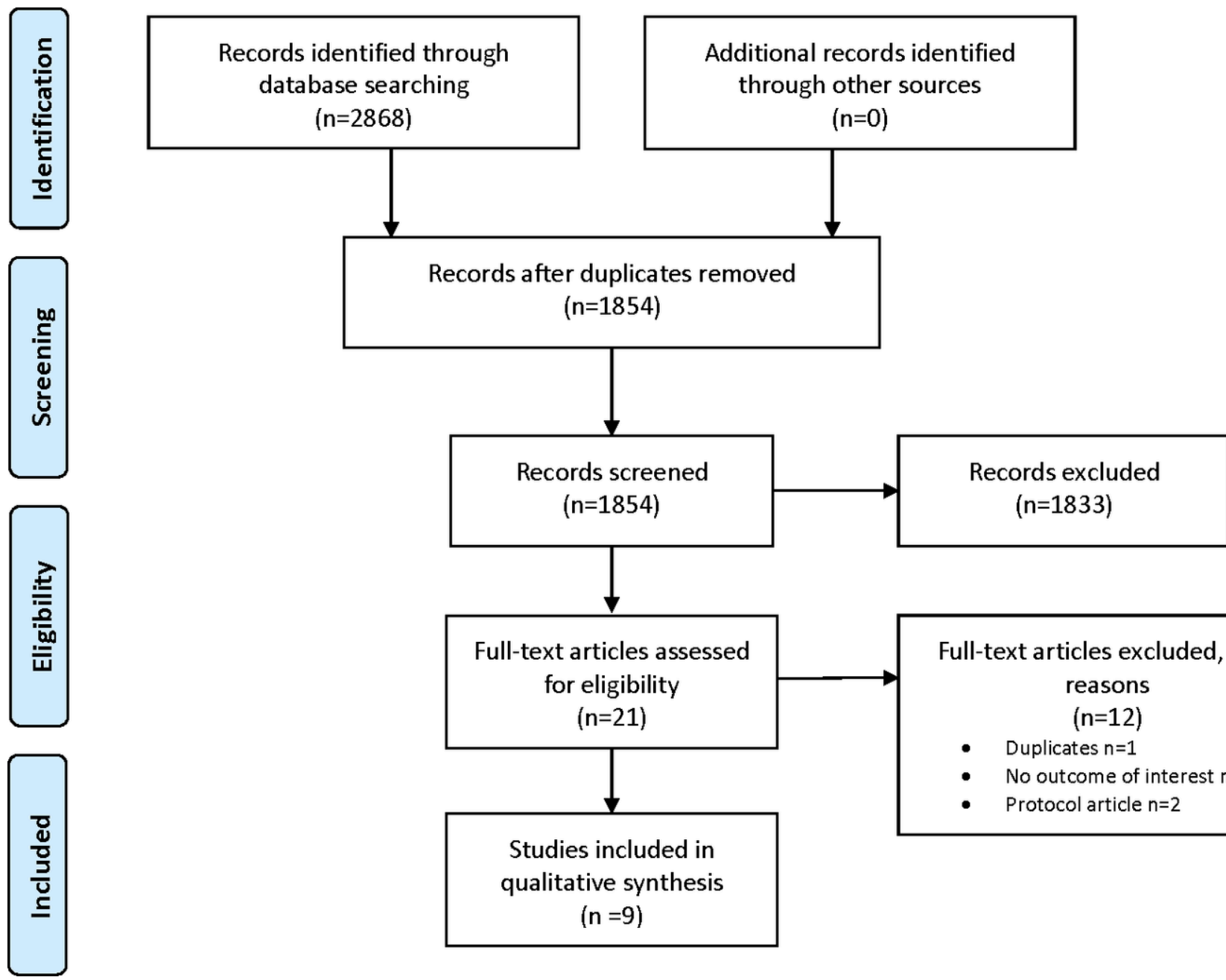

Additional records identified through other sources

$(n=0)$

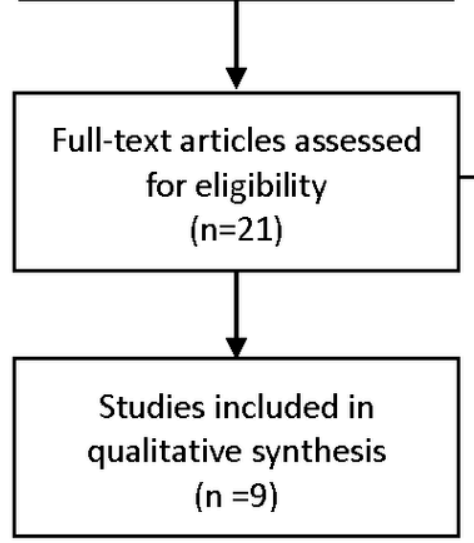

Full-text articles excluded, with

reasons

$(n=12)$

- Duplicates $n=1$

- No outcome of interest $n=9$

- Protocol article $n=2$

Figure 1 Flow chart of the included studies in the systematic reviews. 
Table 1 Studies included in the qualitative synthesis

\begin{tabular}{|c|c|c|c|c|c|c|}
\hline \multicolumn{2}{|l|}{ Study characteristics } & \multicolumn{5}{|l|}{ Participant characteristics } \\
\hline Author and year & Study location & Inclusion criteria & $\begin{array}{l}\text { Participants } \\
\text { included (IG/CG) }\end{array}$ & Women \% & $\begin{array}{l}\text { Age (year) } \\
\text { Mean (SD) }\end{array}$ & $\begin{array}{l}\text { BMI }\left(\mathrm{kg} / \mathrm{m}^{2}\right) \\
\text { Mean (SD) }\end{array}$ \\
\hline Armagan et $a l^{34} 2015$ & Eskisehir, Turkey & With OA (ACR criteria) & $30 / 40$ & $68 \%$ & $56(0.6)$ & $30.9(0.2)$ \\
\hline Dincer et $a l^{\beta 2} 2016$ & Istanbul, Turkey & With OA (ACR criteria) & $19 / 16$ & $80 \%$ & $51(2.4)$ & $28.6(0.8)$ \\
\hline Henriksen et a $\left.\right|^{35} 2014$ & Copenhagen, Denmark & $\begin{array}{l}\text { With } \mathrm{OA} \text { (osteophytes and/or joint space narrowing } \\
\text { assessed by a radiologist) }\end{array}$ & $59 / 63$ & - & $64(0.8)$ & $37.2(0.7)$ \\
\hline Hunter et a $P^{335} 2015$ & North Carolina, USA & $\begin{array}{l}\text { With OA (RKOA KL } 2 \text { or } 3, \mathrm{BMI} \text { of } 27 \text { to } 37 \text { and } \\
\text { sedentary ( }<30 \text { min exercise/week in the past } \\
6 \text { months) }\end{array}$ & $36 / 33$ & $72 \%$ & $66(6)$ & $33.6(3.7)$ \\
\hline Landsmeer et al ${ }^{36} 2016$ & Rotterdam, Holland & $\begin{array}{l}\text { Risk of OA (overweight/obese with no clinical knee } \\
\text { OA according to ACR criteria) }\end{array}$ & $87 / 87$ & $100 \%$ & $56(3.2)$ & $32.3(4.2)$ \\
\hline $\begin{array}{l}\text { Multanen et } a^{\beta 7} 2014 \text { and } \\
\text { Koli et } a l^{40} 2015\end{array}$ & Jyväskyla, Finland & With OA (symptomatic and RKOA KL 1 or 2) & $40 / 40$ & $100 \%$ & $58(4.2)$ & 26.9 \\
\hline Munukka et a $\left.\right|^{38} 2016$ & Jyväskyla, Finland & With OA (symptomatic and RKOA KL 1 or 2) & $43 / 44$ & $100 \%$ & $64(2)$ & $27(0.3)$ \\
\hline Ochiai et al ${ }^{39} 2014$ & Chiba, Japan & With OA (RKOA KL 1, 2, 3) & 9/11 & $100 \%$ & $59(0.7)$ & $22.7(1)$ \\
\hline Roos and Dahlberg 222005 & Malmö,Sweden & Risk of OA (patients having had meniscectomy) & $22 / 23$ & $33 \%$ & $46(3.3)$ & $26.6(3.2)$ \\
\hline
\end{tabular}

$\mathrm{ACR}$, American College of Rheumatology ${ }^{48}$; BMI, body mass index; CG, control group; IG, intervention group; KL, Kellgren-Lawrence scale; OA, osteoarthritis; $\mathrm{P}$, patella; RKOA, radiographic knee osteoarthritis.

Two study comparisons investigated the effect of knee joint loading exercise in participants at increased risk of developing OA: one in participants having had arthroscopic partial meniscectomy ${ }^{28}$ and the other in overweight or obese participants. $^{22} 36$ Twelve study comparisons focused on participants with OA. ${ }^{32-35} 37-40$

\section{Participant characteristics}

The overall number of participants in the included studies was 702 , with a mean age (SD) of 57.7 years (6.5) and a mean BMI $\left(\mathrm{kg} / \mathrm{m}^{2}\right)(\mathrm{SD})$ of 29.5 (4.4). The overall percentage of women was $81.7 \%$ (table 1).

\section{Outcome measures}

In the two study comparisons including participants at risk of $\mathrm{OA}$, articular cartilage was assessed as cartilage morphology using the semiquantitative MRI Osteoarthritis Knee Score (MOAKS) scoring system, ${ }^{36}$ and cartilage composition as GAG via dGEMRIC index. ${ }^{22}$

In the 12 study comparisons focusing on participants with established OA, articular cartilage was assessed using cartilage morphometry in four ${ }^{32}$ and morphology with semiquantitative scoring systems in three. ${ }^{33-35}$ Cartilage composition was assessed in seven comparisons as GAG via dGEMRIC ${ }^{37} 38$ or collagen via T2 mapping. ${ }^{37-40}$

Detailed characteristics of participants and outcome measure characteristics are reported in table 2.

\section{Knee joint loading exercise interventions}

Knee joint loading exercise interventions differ substantially among studies. All but one of the included trials tested the effect of a therapeutic exercise programme. One trial tested the effect from a general physical activity programme in which participants were encouraged to take part in physical activity classes, for example, Nordic walking, volleyball or modern dance. ${ }^{36}$ Furthermore, all the included studies compared a knee joint loading exercise intervention to a non-exercising control group treatment such as local heat or oral glucosamine. Detailed characteristics of knee joint loading exercise interventions are reported in table 2 .

\section{Narrative synthesis of results}

Meta-analysis was not considered appropriate because of the substantial heterogeneity between study interventions, patient characteristics and outcome variables. ${ }^{41}$ Instead, we summarised the results of these studies narratively to provide a clear critical appraisal of the evidence, as recommended by the guidelines on the conduct of narrative synthesis in systematic reviews. ${ }^{42}$

Between-group difference in participants at risk of $O A$

In the participants at risk of OA, one study comparison in overweight women with a mean age of 56 years reported no effect on cartilage defects (MOAKS) ${ }^{36}$ and one in mostly men with a mean age of 46 years, having had arthroscopic partial meniscectomy, reported positive cartilage composition changes on GAG as assessed from dGEMRIC. ${ }^{22}$

Between-group difference in participants with established OA In participants with established OA, six study comparisons found no effect of knee joint loading exercise on cartilage thickness, volume or defects, ${ }^{32-35}$ one study comparison reported no effect ${ }^{37}$ on GAG and one reported a negative effect on the cartilage composition of the medial condyle of the femur, both assessing GAG via dGEMRIC. ${ }^{38}$ On the contrary, the same knee joint loading exercise intervention that reported negative effects on GAG also reported a positive effect on collagen assessed using T2 mapping in the cartilage of the posterior medial femoral condyle and central medial tibial condyle. ${ }^{38}$ Two publications from the same RCT reported a positive effect on collagen T2mapping in the patellar cartilage $e^{40}$ and no effect on the cartilage of the medial condyle of the femur. ${ }^{37}$ Lastly, one study comparison reported no effect ${ }^{3739}$ on collagen T2 mapping ${ }^{39}$ (table 3).

\section{Within-group difference}

The within-group differences analysis investigating articular cartilage changes preintervention to postintervention (within-group findings) showed that knee joint loading exercise increased cartilage volume ${ }^{32}$ and had a positive effect on cartilage defects in the medial femoral condyle ${ }^{34}$ and on GAG in the medial and lateral compartment of the femur and lateral compartment of the tibia. ${ }^{22} 37$ Furthermore, positive effects were also reported 


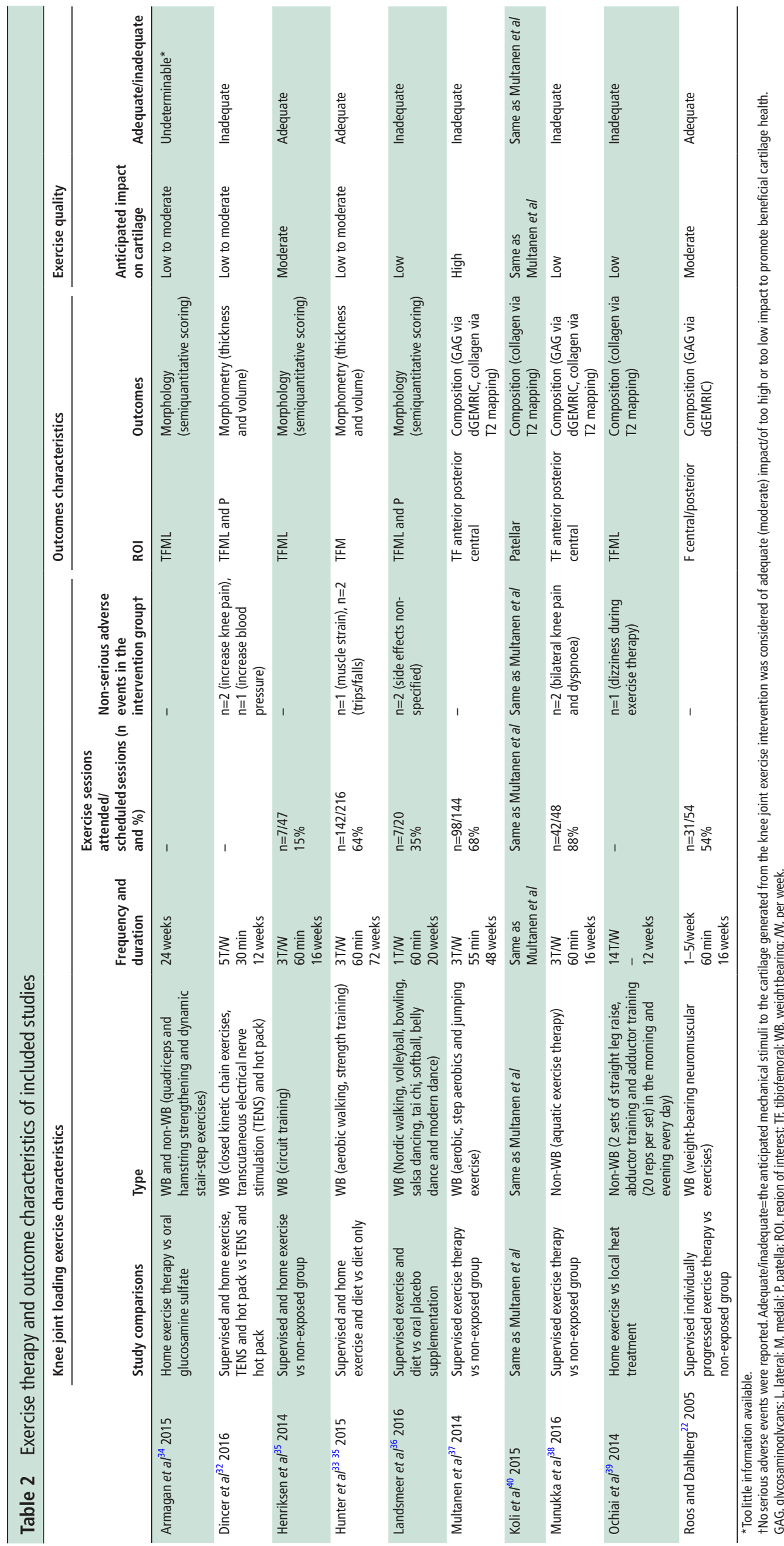




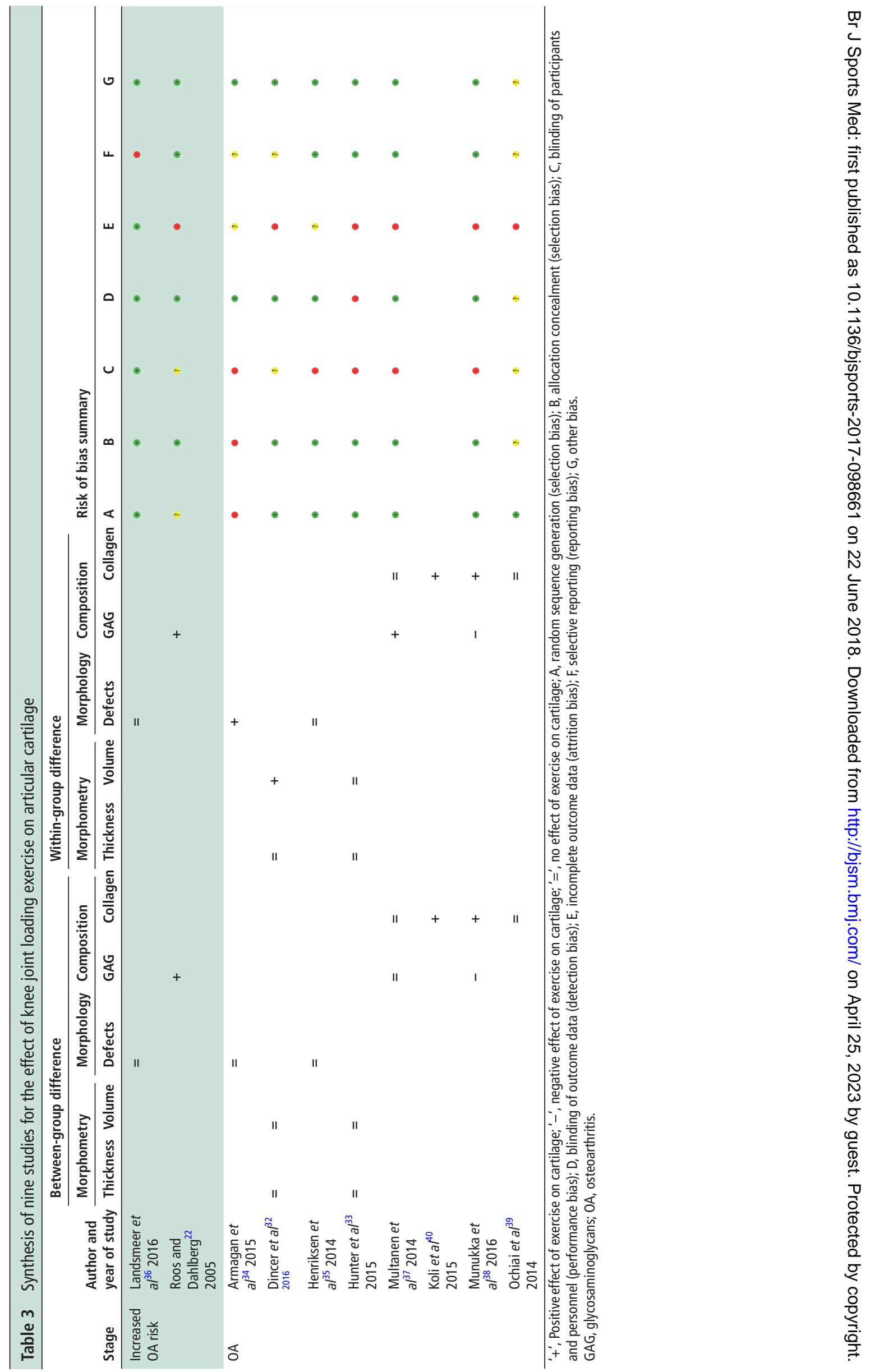


on the patellar cartilage ${ }^{40}$ and on the posterior medial femoral condyle and central medial tibial condyle. ${ }^{38}$ There was only one negative within-group finding out of 14 comparisons.

\section{Subgroup analysis on cartilage compartment}

Three out of nine studies assessed the effect of knee joint loading exercise on the patellar compartment in addition to the tibiofemoral compartment. ${ }^{32} 3640$ In one study, ${ }^{36}$ the patellar and tibiofemoral compartments were combined for the assessment of exercise on cartilage health, not allowing for comparisons of different cartilage compartments. In contrast, two studies ${ }^{32} 40$ analysed the patellar and tibiofemoral compartments separately. One study reported a beneficial effect on the collagen matrix in the patellar but not in the tibiofemoral compartment, ${ }^{40}$ and another study reported no effect in cartilage volume or thickness for the patellar and tibiofemoral compartment. ${ }^{32}$

\section{Impact of sex on cartilage health}

We found no indication of difference in the effect of exercise on cartilage health between the sexes. Four studies, seven study comparisons, included only women, of which two study comparisons reported a positive effect on collagen, ${ }^{38} 40$ one reported a negative effect on $\mathrm{GAG}^{38}$ and four reported no effect of knee joint loading exercise on cartilage health. ${ }^{36} 3739$

Five studies, seven study comparisons, included both men and women, of which one reported a beneficial effect on $\mathrm{GAG}^{22}$ and six reported no effect of knee joint loading exercise on cartilage health (table 3). ${ }^{32} 3435$

\section{Quality of evidence \\ Risk of bias}

Overall, the majority of the studies applied proper randomisation, allocation and blinding of the outcome assessment. In contrast, all the studies failed to clearly report, or inadequately addressed, dropouts of participants in the analyses (attrition bias, table 3 ).

\section{Knee joint loading exercise quality}

When evaluated and rated independently by two of the coauthors (CBJ and EMR), some of the exercise interventions were assessed as including activities being considered outside the therapeutic loading spectrum and therefore not necessarily adequate to promote positive articular cartilage (table 2). This classification was purely done for descriptive purposes, and the number of studies did not allow for subgroup analyses.

\section{GRADE assessment}

The inadequacy of some knee joint loading interventions, the small number of studies and the few participants involved limit the generalisability of our findings. Therefore, due to this indirectness and imprecision, the overall quality of evidence was deemed low (online supplementary appendix C).

\section{DISCUSSION}

Our findings suggest that knee joint loading exercise seems not to be harmful for articular cartilage in people at increased risk of, or with, knee OA. However, the quality of evidence was low.

\section{Articular cartilage morphometry and morphology}

The inconclusive findings about knee joint loading and the impact on cartilage thickness, volume and defects may relate to the heterogeneity of the populations, the interventions studied or the outcomes used. In fact, when evaluated and rated independently by two of the coauthors (CBJ and EMR), not all the exercise interventions were assessed as adequate to promote positive articular cartilage changes. In some cases, the dose was considered too low, and in one case, the type of exercise (jumps) was considered excessive for the cartilage of older women who had mild OA. Additionally, the compliance with the exercise interventions investigating cartilage morphometry or morphology was generally poor. The resulting inadequate mechanical stimuli could potentially be at least partly responsible for the lack of effect. On the other hand, MRI-based cartilage assessments have been shown to be sensitive enough to detect between-group morphometry and morphology changes in previous randomised studies using quantitative and semiquantitative methods. ${ }^{43}$ Nevertheless, in our review, the studies assessing cartilage with both quantitative and semiquantitative methods failed to report a change for either method, suggesting the lack of positive effect was not due to poor responsiveness of the evaluation methods.

\section{Articular cartilage composition}

It is well known that alterations in articular cartilage composition is a marker of early OA changes. ${ }^{44}$ Negative changes in cartilage composition may therefore be expected to occur prior to changes in morphometry and morphology cartilage parameters. ${ }^{45}$ None of the studies included in our review allowed for a comparison of treatment effects on both structural and compositional changes of the cartilage. However, GAG and collagen assessed as dGEMRIC and T2 mapping, respectively, were the only outcomes that showed a response to the treatment interventions, supporting the theory that these early OA markers are sufficiently sensitive to detect treatment effects in individuals with early or established OA. Nevertheless, six out of seven study comparisons found no effect or beneficial effect or beneficial effect on cartilage composition, highlighting that knee joint loading exercise seems to be at least safe in patients at increased risk of, or with, knee OA.

\section{Limitations}

This study has some limitations. The heterogeneity of the interventions, patient characteristics and outcome variables did not support the use of a meta-analysis. Instead, in accordance with the Cochrane Handbook, we described our findings narratively. ${ }^{23}$ Although, from a statistical point of view, there is no restriction on study number or similarity, it is important to consider the conceptual diversity of the included studies for the meta-analysis to be meaningful for researchers, clinicians and patients. ${ }^{41}$ Furthermore, the low compliance with the exercise interventions in studies investigating articular cartilage morphology and morphometry limits the possibility of concluding whether exercise had a positive or negative impact on these outcome measures. Additionally, the included studies did not allow for comparison of different exercise programmes and/or comparisons of specific cartilage compartments since all studies included a non-exercising control arm and only two studies reported the patellofemoral compartment separately. Thus, our findings are restricted to the effect of increased knee joint loading from therapeutic exercise compared with no change in knee joint loading, particularly in the tibiofemoral compartment. As no meta-analysis was performed, precision, inconsistency and publication bias were based on the narrative synthesis of results. Finally, one trial included the control treatment of glucosamine ${ }^{34}$ and another trial included a control of local heat treatment. ${ }^{39}$ Recent systematic reviews conclude that glucosamine does not impact cartilage 
health, ${ }^{4647}$ and there is no evidence to suggest an effect of local heat treatment on articular cartilage.

\section{Implications for researchers and clinicians}

More high-quality RCTs are needed to further investigate the impact of knee joint loading exercise on articular cartilage in patients at increased risk of, or with, knee OA. To increase the possibility of finding positive effects, available results suggest future studies need to focus on interventions in the form of supervised weight-bearing exercise therapy of sufficient dose in younger subjects at risk or in early stages of the disease, allowing for evaluation of cartilage composition with measures such as dGEMRIC and T2 mapping.

\section{CONCLUSION}

We narratively summarised the impact of knee joint loading exercise on knee joint articular cartilage in the participants at risk of, or with, knee OA included in RCTs of exercise. Knee joint loading exercise seems not to harm articular cartilage in participants at increased risk of, or with, knee OA. However, the quality of evidence was low, including some interventions studying activities considered outside the therapeutic loading spectrum to promote cartilage health.

\section{What is already known?}

- Knee joint loading exercise is a cornerstone in the management of knee osteoarthritis (OA).

- Knee joint loading exercise in the form of exercise therapy has a moderate effect in reducing pain and improving physical function in patients with knee OA.

\section{What are the new findings?}

- Knee joint loading exercise seems to not be harmful for articular cartilage in participants at increased risk of, or with, knee OA.

- Knee joint loading exercise interventions at a dose sufficient to improve cartilage health need to be investigated.

Contributors Conception or design of the work: $A B, C B J, M S, W W$, EMR. Data collection: $A B, C B J$. Data analysis and interpretation: $A B, C B J, M S, W W$, EMR. Drafting the article: $A B$. Critical revision of the article: $A B, C B J, M S, W W$, EMR. Final approval of the version to be published: $A B, C B J, M S, W W, E M R$.

Funding This project is supported by a European Union Seventh Framework Programme (FP7-PEOPLE-2013-ITN; KNEEMO) under grant agreement number 607510.

Disclaimer The funding source had no involvement in any aspect of this systematic review.

\section{Competing interests None declared.}

Patient consent Not required.

Provenance and peer review Not commissioned; externally peer reviewed.

(c) Article author(s) (or their employer(s) unless otherwise stated in the text of the article) 2019. All rights reserved. No commercial use is permitted unless otherwise expressly granted.

\section{REFERENCES}

1 Cross M, Smith E, Hoy D, et al. The global burden of hip and knee osteoarthritis: estimates from the Global Burden of Disease 2010 study. Ann Rheum Dis 2014;73:1323-30

2 Wallace IJ, Worthington S, Felson DT, et al. Knee osteoarthritis has doubled in prevalence since the mid-20th century. Proc Natl Acad Sci U SA 2017;114:9332-6.

3 Vos T, Flaxman AD, Naghavi M, et al. Years lived with disability (YLDs) for 1160 sequelae of 289 diseases and injuries 1990-2010: a systematic analysis for the Global Burden of Disease Study 2010. Lancet 2012;380:2163-96.
4 Salmon JH, Rat AC, Sellam J, et al. Economic impact of lower-limb osteoarthritis worldwide: a systematic review of cost-of-illness studies. Osteoarthritis Cartilage 2016;24:1500-8.

5 Felson DT. Osteoarthritis as a disease of mechanics. Osteoarthritis Cartilage 2013;21:10-15.

6 Robinson WH, Lepus CM, Wang Q, et al. Low-grade inflammation as a key mediator of the pathogenesis of osteoarthritis. Nat Rev Rheumatol 2016;12:580-92.

7 Buckwalter JA. Sports, joint injury, and posttraumatic osteoarthritis. J Orthop Sports Phys Ther 2003;33:578-88.

8 Muthuri SG, McWilliams DF, Doherty M, et al. History of knee injuries and knee osteoarthritis: a meta-analysis of observational studies. Osteoarthritis Cartilage 2011;19:1286-93.

9 Lohmander LS, Ostenberg A, Englund M, et al. High prevalence of knee osteoarthritis, pain, and functional limitations in female soccer players twelve years after anterior cruciate ligament injury. Arthritis Rheum 2004:50:3145-52.

10 Brown TD, Johnston RC, Saltzman CL, et al. Posttraumatic osteoarthritis: a first estimate of incidence, prevalence, and burden of disease. J Orthop Trauma 2006;20:739-44

11 Quicke JG, Foster NE, Thomas MJ, et al. Is long-term physical activity safe for older adults with knee pain?: a systematic review. Osteoarthritis Cartilage 2015;23:1445-56

12 Juhl C, Christensen R, Roos EM, et al. Impact of exercise type and dose on pain and disability in knee osteoarthritis: a systematic review and meta-regression analysis of randomized controlled trials. Arthritis Rheumatol 2014:66:622-36.

13 McAlindon TE, Bannuru RR, Sullivan MC, et al. OARSI guidelines for the non-surgical management of knee osteoarthritis. Osteoarthritis Cartilage 2014;22:363-88.

14 Roos EM. Osteoarthritis 2012 year in review: rehabilitation and outcome year in review: rehabilitation and outcomes. Osteoarthritis and cartilage / OARS, Osteoarthritis Research Society 2012:20:1477-83.

15 Fernandes GS, Parekh SM, Moses J, et al. Prevalence of knee pain, radiographic osteoarthritis and arthroplasty in retired professional footballers compared with men in the general population: a cross-sectional study. Br I Sports Med 2018;52.

16 Tveit M, Rosengren BE, Nilsson JÅ, et al. Former male elite athletes have a higher prevalence of osteoarthritis and arthroplasty in the hip and knee than expected. Am J Sports Med 2012;40:527-33.

17 Jørgensen AEM, Kjær M, Heinemeier KM. The effect of aging and mechanical loading on the metabolism of articular cartilage. J Rheumatol 2017;44:410-7.

18 Roos EM, Arden NK. Strategies for the prevention of knee osteoarthritis. Nat Rev Rheumatol 2016;12:92-101

19 Munukka M, Waller B, Häkkinen A, et al. Physical activity is related with cartilage quality in women with knee osteoarthritis. Med Sci Sports Exerc 2017:49:1323-30

20 Lin W, Alizai H, Joseph GB, et al. Physical activity in relation to knee cartilage T2 progression measured with 3 T MRI over a period of 4 years: data from the Osteoarthritis Initiative. Osteoarthritis Cartilage 2013:21:1558-66.

21 Wondrasch B, Risberg MA, Zak L, et al. Effect of accelerated weightbearing after matrix-associated autologous chondrocyte implantation on the femoral condyle: a prospective, randomized controlled study presenting MRI-based and clinical outcomes after 5 years. Am J Sports Med 2015:43:146-53.

22 Roos EM, Dahlberg L. Positive effects of moderate exercise on glycosaminoglycan content in knee cartilage: a four-month, randomized, controlled trial in patients at risk of osteoarthritis. Arthritis \& Rheumatism 2005;52:3507-14.

$23 \mathrm{JPea} \mathrm{H}$. Cochrane handbook for systematic reviews of interventions version 5.1.0 (updated March 2011): The Cochrane Collaboration:2011 2011.

24 Kellgren JH, Lawrence JS. Radiological assessment of osteo-arthrosis. Ann Rheum Dis 1957:16:494-502.

25 Caspersen CJ, Powell KE, Christenson GM. Physical activity, exercise, and physical fitness: definitions and distinctions for health-related research. Public Health Rep 1985;100:126-31.

26 de Vries RB, Hooijmans CR, Tillema A, et al. Updated version of the Embase search filter for animal studies. Lab Anim 2014;48:88.

27 Hooijmans CR, Tillema A, Leenaars $M$, et al. Enhancing search efficiency by means of a search filter for finding all studies on animal experimentation in PubMed. Lab Anim 2010:44:170-5.

28 Lammentausta E, Kiviranta P, Nissi MJ, et al. T2 relaxation time and delayed gadolinium-enhanced MRI of cartilage (dGEMRIC) of human patellar cartilage at 1.5 T and 9.4 T: relationships with tissue mechanical properties. J Orthop Res 2006;24:366-74

29 Nieminen MT, Rieppo J, Töyräs J, et al. T2 relaxation reveals spatial collagen architecture in articular cartilage: a comparative quantitative MRI and polarized light microscopic study. Magn Reson Med 2001:46:487-93.

30 Eckstein F, Lemberger B, Gratzke C, et al. In vivo cartilage deformation after different types of activity and its dependence on physical training status. Ann Rheum Dis 2005;64:291-5

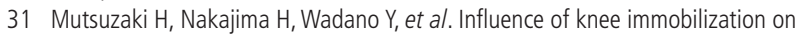
chondrocyte apoptosis and histological features of the anterior cruciate ligament insertion and articular cartilage in rabbits. Int J Mol Sci 2017;18:253. 
32 Dinçer Ümit, et al. The effects of closed kinetic chain exercise on articular cartilage morphology: myth or reality? A randomized controlled clinical trial. Türkiye Fiziksel Tip ve Rehabilitasyon Dergisi 2016;62:28-36.

33 Hunter DJ, Beavers DP, Eckstein F, et al. The Intensive Diet and Exercise for Arthritis (IDEA) trial: 18-month radiographic and MRI outcomes. Osteoarthritis Cartilage 2015;23:1090-8.

34 Armagan O, Yilmazer S, Calısir C, et al. Comparison of the symptomatic and chondroprotective effects of glucosamine sulphate and exercise treatments in patients with knee osteoarthritis. J Back Musculoskelet Rehabil 2015;28:287-93.

35 Henriksen M, Christensen R, Hunter DJ, et al. Structural changes in the knee during weight loss maintenance after a significant weight loss in obese patients with osteoarthritis: a report of secondary outcome analyses from a randomized controlled trial. Osteoarthritis Cartilage 2014;22:639-46.

36 Landsmeer ML, Runhaar J, van der Plas P, et al. Reducing progression of knee OA features assessed by MRI in overweight and obese women: secondary outcomes of a preventive RCT. Osteoarthritis Cartilage 2016;24:982-90.

37 Multanen J, Nieminen MT, Häkkinen A, et al. Effects of high-impact training on bone and articular cartilage: 12-month randomized controlled quantitative MRI study. J Bone Miner Res 2014:29:192-201.

38 Munukka M, Waller B, Rantalainen T, et al. Efficacy of progressive aquatic resistance training for tibiofemoral cartilage in postmenopausal women with mild knee osteoarthritis: a randomised controlled trial. Osteoarthritis Cartilage 2016;24:1708-17.

39 Ochiai S, Watanabe $\mathrm{A}, \mathrm{Oda} \mathrm{H}$, et al. Effectiveness of thermotherapy using a heat and steam generating sheet for cartilage in knee osteoarthritis. J Phys Ther Sci 2014;26:281-4.
40 Koli J, Multanen J, Kujala UM, et al. Effects of exercise on patellar cartilage in women with mild knee osteoarthritis. Med Sci Sports Exerc 2015;47:1767-74.

41 Matthias Egger GDS, Altman DG. Systematic Reviews in Health Care: Meta-Analysis in Context. 2nd edn: BMJ Publishing Group, 2008.

42 Popay JRH, Sowden A, Petticrew M, et al. Guidance on the conduct of narrative synthesis in systematic reviews. a product from the ESRC methods programme. ESRC 2006.

43 Lohmander LS, Hellot S, Dreher D, et al. Intraarticular sprifermin (recombinant human fibroblast growth factor 18) in knee osteoarthritis: a randomized, double-blind, placebo-controlled trial. Arthritis Rheumatol 2014;66:1820-31.

44 Migliore A, Massafra U. Towards the identification of early stage osteoarthritis. Clin Cases Miner Bone Metab 2014;11:114-6.

45 Guermazi A, Alizai H, Crema MD, et al. Compositional MRI techniques for evaluation of cartilage degeneration in osteoarthritis. Osteoarthritis Cartilage 2015;23:1639-53.

46 Wandel S, Jüni P, Tendal B, et al. Effects of glucosamine, chondroitin, or placebo in patients with osteoarthritis of hip or knee: network meta-analysis. BMJ 2010;341:c4675.

47 Towheed TE, Maxwell L, Anastassiades TP, et al. Glucosamine therapy for treating osteoarthritis—art. no. CD002946.pub2. Cochrane Db Syst Rev 2005

48 Altman R, Asch E, Bloch D, et al. Development of criteria for the classification and reporting of osteoarthritis. Classification of osteoarthritis of the knee. Diagnostic and Therapeutic Criteria Committee of the American Rheumatism Association. Arthritis Rheum 1986;29:1039-49. 